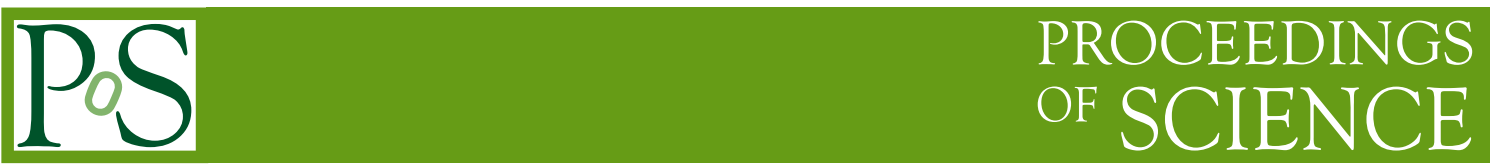

\title{
Magnetic field damping in the Vela Jr. SNR
}

\author{
lurii Sushch* \\ DESY, D-15738 Zeuthen, Germany \\ Astronomical Observatory of Ivan Franko National University of L'viv, vul. Kyryla i Methodia, \\ 8, L'viv 79005, Ukraine \\ E-mail: iurii.sushch@desy.de
}

Robert Brose and Martin Pohl

DESY, D-15738 Zeuthen, Germany

Institute of Physics and Astronomy, University of Potsdam, 14476, Potsdam, Germany

Vela Jr. (RX J0852.0-4622) is one of just a few known supernova remnants (SNRs) with the resolved shell across the whole electromagnetic spectrum from radio to very-high-energy ( $>100$ $\mathrm{GeV}$; VHE) gamma-rays. Its proximity and large size allow for detailed spatially resolved observations of the source making Vela Jr. one of the primary sources used for the study of particle acceleration and emission mechanisms in SNRs. High-resolution X-ray observations reveal steepening of the spectrum towards the interior of the remnant. In this study we aim for the self-consistent radiation model of Vela Jr. which at the same time would explain the broadband emission from the source and its spatially-dependent features. We solve the full particle transport equation combined with the high-resolution 1D hydrodynamic simulations (using Pluto code) and subsequently calculate the radiation from the remnant. We show that accounting for the damping of strong magnetic turbulence downstream of the forward shock might explain the observed radial dependence of the X-ray spectrum.

35th International Cosmic Ray Conference - ICRC2017

10-20 July, 2017

Bexco, Busan, Korea

${ }^{*}$ Speaker. 


\section{Introduction}

Supernova remnants (SNRs) are widely considered to be main candidates for the acceleration sites of Galactic cosmic rays (CRs) which places them among the most interesting and most studied astrophysical objects. Vela Jr. is one of just a few SNRs with detected non-thermal emission and well resolved shell-like morphology across the whole electromagnetic spectrum from radio to very-high-energy ( $>100 \mathrm{GeV}$; VHE) gamma-rays. Its proximity, large size and strong non-thermal emission (specifically from the north-western rim of the remnant) allows for detailed spatially resolved observations of the source both in X-rays and at $\mathrm{TeV}$ energies making it one of the prime sources for studies of particle acceleration and radiation mechanisms in SNRs.

High resolution X-ray observations reveal thin and bright filamentary structures in the north western part of the shell (Bamba et al., 2005). These filaments are usually explained by fast synchrotron cooling in strongly amplified magnetic field $(B \gtrsim 100 \mu \mathrm{G}$; see e.g. Berezhko et al., 2009). However, it is also possible that they are limited by magnetic field damping downstream the forward shock (Pohl et al., 2005; Rettig \& Pohl, 2012). In this scenario strong magnetic turbulence formed upstream is damped downstream at typical length scales comparable to the width of the filaments. Recently a detailed analysis of the archival XMM-Newton data revealed a gradual softening of the spectrum (from a photon index of 2.56 to a photon idnex of 2.96) in the north western rim towards the interior of the remnant (Kishishita et al., 2013). This softening was interpret by the change in the cut-off energy due to synchrotron cooling. Using a simple spectral evolution model assuming that the downstream time evolution of the maximum electron energy is determined only by synchrotron losses, Kishishita et al. (2013) showed that the softening of the spectrum can be explained by a rather low magnetic field of $\sim 5-20 \mu \mathrm{G}$. This would require however a quite high electron maximum energy of about $\sim 100 \mathrm{TeV}$ which is in contradiction with H.E.S.S. observations at $\mathrm{TeV}$ energies (H. E. S. S. Collaboration et al., 2016).

In this paper we provide modeling of the non-thermal emission from the Vela Jr. SNR focusing on explaining radial profile properties of the remnant. In this regard we consider two scenarios depending on the effect which determines the radial change of the spectrum: magnetic field damping scenario and the synchrotron cooling scenario.

\section{Modelling}

\subsection{Hydrodynamics}

We model the hydrodynamic evolution of the Vela Jr. SNR with high resolution one-dimensional (1D) simulations using Pluto code (Mignone et al., 2012). We assume that Vela Jr. was created in a core collapse Supernova ( $\mathrm{SN}$ ) explosion which is supported by the detection of the central compact object (CCO) AX J0851.9-4617.4 ${ }^{1}$ close to the center of the remnant (Aschenbach, 1998; Aschenbach et al., 1999; Slane et al., 2001). However, it is not clear whether the association with the SNR is correct because no pulsations were detected and later it was also suggested that a potential CCO might be an unrelated planetary nebula (Reynoso et al., 2006). Additionaly, the absence of broad Ca II absorption lines in spectra of background stars together with the constrains set by the

\footnotetext{
${ }^{1}$ Also known as CXOU J085201.4-461753
} 
Table 1: Parameters of the hydrodynamic model

\begin{tabular}{lc}
\hline \hline Parameter & Value \\
\hline$M_{\star}\left[M_{\odot}\right]$ & 3 \\
$v_{\text {wind }}[\mathrm{km} / \mathrm{s}]$ & 15 \\
$\dot{M}_{\star}\left[M_{\odot} / \mathrm{yr}\right]$ & $1.2 \times 10^{-5}$ \\
$R_{\mathrm{sh}}[\mathrm{pc}]$ & 13.24 \\
$V_{\mathrm{sh}}[\mathrm{km} / \mathrm{s}]$ & 2970 \\
$n_{0}\left[\mathrm{~cm}^{-3}\right]$ & 0.014 \\
$T[\mathrm{yr}]$ & 3300 \\
$D[\mathrm{pc}]$ & 750 \\
\hline
\end{tabular}

$\mathrm{Ti}^{44}$ gamma-ray line also suggests a core collapse origin with SNe types of Ic or Ibc (Iyudin et al., 2010).

The density of the ambient medium is constrained by the lack of the X-ray thermal emission which places the upper limit at $n_{0}<2.9 \times 10^{-2}\left(\frac{D}{1 \mathrm{kpc}}\right)^{-1 / 2} f^{-1 / 2} \mathrm{~cm}^{-3}$, where $D$ is the distance to the remnant and $f$ is the volume filling factor (Slane et al., 2001). It should be noted however that the lack of thermal emission can be claimed only for the gas with a temperature above $1 \mathrm{keV}$, because at energies below $1 \mathrm{keV}$ the observed $\mathrm{X}$-ray emission is dominated by the strong thermal emission from the Vela SNR. In our hydrodynamic simulations we assume that the SNR is expanding into a wind zone, i.e. the density radial distribution follows $n \propto r^{-2}$, and require the current upstream density to be lower than $0.03 \mathrm{~cm}^{-3}$.

The distance to the remnant and its age can be constrained by its angular size and its angular expansion rate measured by Katsuda et al. (2008) and Allen et al. (2015) using XMM-Newton and Chandra data respectively. XMM-Newton data taken between 2001 and 2007 implies an expansion rate of $0.84^{\prime \prime} \pm 0.23^{\prime \prime} \mathrm{yr}^{-1}$ (Katsuda et al., 2008) while Chandra data from 2003 to 2008 suggests an expansion rate of $0.42^{\prime \prime} \pm 0.10^{\prime \prime} \mathrm{yr}^{-1}$ Allen et al. (2015). In our simulations we use the measurement provided by XMM-Newton to constrain our model.

We tested several models assuming different values for the mass of the star before the explosion and fixing the explosion energy at $10^{51} \mathrm{erg}$. We stop our simulations at the moment when the ratio of the expansion rate to the radius of the remnant is equal to the measured value. The parameters for the hydrodynamic model which we picked to use for our simulations of the particle acceleration and non-thermal radiation are collected in the Table 1.

\subsection{Magnetic field}

We assume that the interstellar magnetic field $B_{\mathrm{ISM}}$ is amplified upstream of the shock by streaming instabilities. The magnetic field strength in the immediate upstream region is then given by $B_{\mathrm{u}}=k B_{\mathrm{ISM}}$. Then for the shock compression ratio of 4 the immediate downstream magnetic field strength is $B_{\mathrm{d}}=\sqrt{11} B_{\mathrm{u}}$.

We consider two different models to describe the distribution of the magnetic field downstream the shock. In the magnetic field damping scenario we assume that turbulently amplified magnetic field is effectively damped downstream of the forward shock of the remnant. The magnetic turbu- 
lence created upstream and at the shock by various instabilities is transfered downstream where it is eventually damped due to the lack of the turbulence growth (Pohl et al., 2005). To describe the magnetic field profile downstream the shock we adopt a simple parametrisation (Pohl et al., 2005)

$$
B(r)=B_{0}+\left(B_{\mathrm{d}}-B_{0}\right) e^{\left(r-R_{\mathrm{sh}}\right) / l_{\mathrm{d}}},
$$

where $B_{0}$ is the magnetic field far downstream of the remnant, $B_{\mathrm{d}}$ is the magnetic field strength in the immediate downstream of the shock, and $l_{\mathrm{d}}$ is the damping length scale.

Alternatively, in the synchrotron cooling model we assume that the immediate downstream magnetic field $B_{\mathrm{d}}$ is transported inside the SNR with the plasma flow and evolved folowing the induction equation for ideal MHD.

\subsection{Particle acceleration}

We simulate the particle density evolution solving the transport equation accounting for synchrotron losses. The spatial diffusion coefficient is assumed to be Bohm-like. Particle injection is determined by the injection parameter $\xi=p_{\text {inj }} / p_{\text {th }}$, where $p_{\text {th }}=\left(2 m k_{\mathrm{B}} T_{\mathrm{d}}\right)$ is the momentum of the particles in thermal peak of the Maxwellian distribution in the downstream plasma with temperature $T_{\mathrm{d}}$ and $p_{\text {inj }}$ is the minimum momentum for which a thermal particle can cross the shock, i.e. thermal particles with momentum $p>p_{\text {inj }}=\xi p_{\text {th }}$ are injected into the acceleration process (Blasi et al., 2005). In our simulations we set $\xi=4.2$. We solve the transport equation separately for electrons and protons as described in (Telezhinsky et al., 2012, 2013) in 1D taking into account only a forward shock and ignoring a reverse shock. The resulting particle spectrum at the current age of the remnant is then used to simulate non-thermal emission.

\subsection{Non-thermal emission}

We calculate non-thermal emission from the remnant considering three emission processes: synchrotron radiation, inverse Compton (IC) scattering of accelerated electrons on the Cosmic Microwave Background (CMB) and proton interactions with subsequent pion decay. For IC scattering we do not consider other possible radiation fields as the Vela Jr. SNR is located far away from the Galactic center and local optical and infrared radiation fields are weak and should not play a strong role. Synchrotron emission is calculated accounting for the turbulent magnetic field (Pohl et al., 2015).

\section{Results}

As mentioned above we adopt two different scenarios to describe the distribution of the magnetic field downstream the shock: magnetic field damping scenario and synchrotron cooling scenario. The choice of the magnetic field distribution model determines the properties of the X-ray emission from the remnant. In the magnetic field damping scenario the flux radial profile and the change of the spectral shape of the X-ray emission with the distance from the shock is determined by the magnetic field strength, while in the synchrotron cooling scenario the radial properties of the $\mathrm{X}$-ray emission are determined by the distribution of the electrons downstream the shock and by synchrotron cooling. Parameters which describe the magnetic field in both scenarios are collected in the Table 2. 
Table 2: Parameters of the magnetic field distribution

\begin{tabular}{l|c|c}
\hline \hline Parameter & Magnetic field damping & Synchrotron cooling \\
\hline$B_{\mathrm{ISM}}, \mu \mathrm{G}$ & 1 & 0.4 \\
$k$ & 4 & 4.7 \\
$B_{0}, \mu \mathrm{G}$ & 1 & - \\
$l_{\mathrm{d}}$ & $0.05 R_{\mathrm{Sh}}$ & - \\
\hline
\end{tabular}

Figure 1 shows the observed broadband spectral energy distribution (SED) overlaid with the curves representing simulated emission in the magnetic field damping scenario (left panel) and synchrotron cooling scenario (right panel). The total emission from the remnant is calculated assuming a spherical symmetry of the remnant. In both scenarios the modelled SED fits the observed X-ray and gamma-ray emission relatively welll, but strongly underpredict the radio emission from the source. This discrepancy is further discussed in the next section.

X-ray radial profiles are calculated following the definition of the regions from Kishishita et al. (2013) for direct comparison of our results with the observational data. Assuming a spherical symmetry we produce energy dependent $2 \mathrm{D}$ flux maps with a cell size of $0.67^{\prime}$, equal to the width of the regions defined for the X-ray spectral analysis in Kishishita et al. (2013). Then for the cells which correspond to the location of the regions we extract the flux and the specrum in the energy range between $2 \mathrm{keV}$ and $10 \mathrm{keV}$. The extracted spectrum from each cell is fit with a simple power law yielding the spectral index distribution (Fig. 2 middle) and with an exponentially cutoff power law assuming the spectral index of 1.6 yielding the cut-off energy distribution (Fig. 2 top). Simulated spatial distributions of the spectral index and of the cut-off energy in the magnetic field damping scenario follow the same trend as the observed radial distributions. However the observed variation of the spectral parameters is stronger. In the case of the synchrotron cooling scenario expected variation of the spectral parameters is very weak, which is mainly due to the low magnetic field strength used in the model. Similarly, the simulated flux profile (Fig. 2 bottom) in the magnetic field damping scenario shows a similar shape with the flux peaking around $0.98 R_{\mathrm{sh}}$ which is not the case in the synchrotron cooling scenario. In both cases, however, the magnitude of the flux decrease cannot be reproduced. It should be noted that both simulated flux profiles are normalized for better comparison with the observational data. Simulated flux is weaker than the observed one, which is because we assume a spherical symmetry while observations were focussed on the brightest region of the SNR.

\section{Discussion}

We strongly underpredict radio emission from Vela Jr. in both cases which have been considered. However, it can be reproduced if we account for other possible processes which might operate in the SNR, e.g. stochastic re-acceleretation of particles at the turbulence in the immediate downstream region of the shock (Pohl et al., 2015). It should also be noted that the reported value of the detected radio flux can be misleading as the remnant is located in a very crowded region with a very complicated background. 

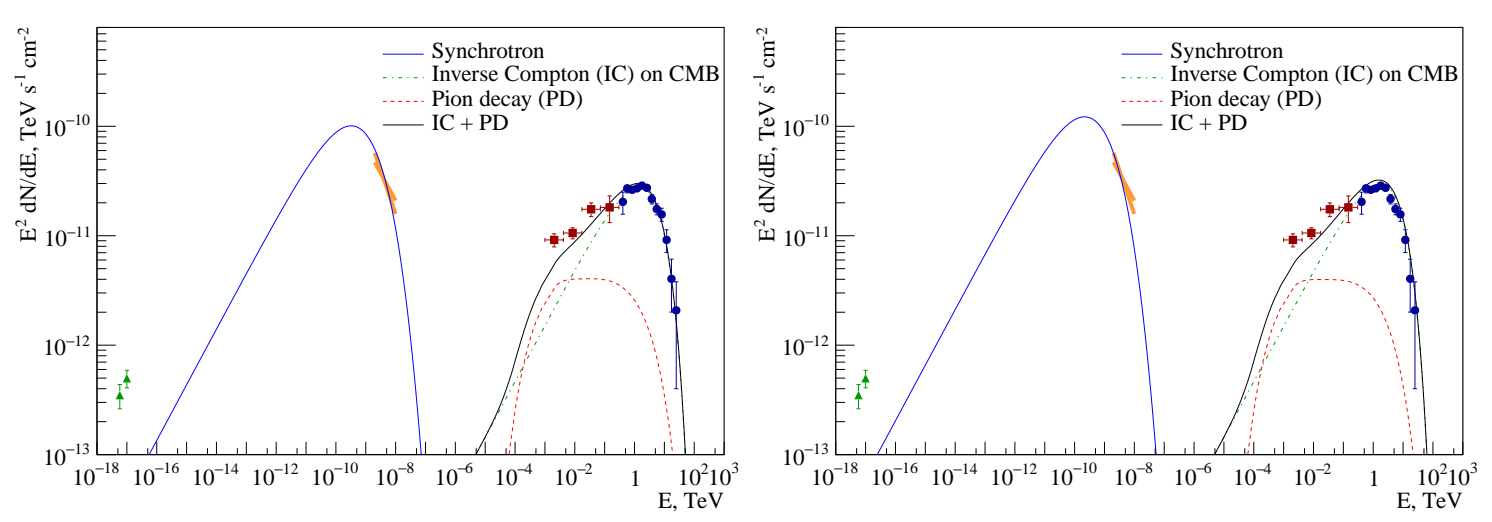

Figure 1: Spectral enery distribution of the Vela Jr. SNR. Lines represent simulated emission from the source produced in different processes as specified in the legend in the magnetic field damping model (left panel) and synchrotron cooling model (right panel). Green triangles indicate the observed radio emission as detected by Parkes (Duncan \& Green, 2000), an orange bow-tie shows the spectral fit of the X-ray XMM-Newton data (Aharonian et al., 2007), red squares represents the Fermi-LAT data points (Tanaka et al., 2011) and blue circles show the H.E.S.S. data points (H. E. S. S. Collaboration et al., 2016).

Magnetic field damping scenario much better reproduces the observed spatial properties of the X-ray emission with respect to the synchrotron cooling scenario. Simultaneous fitting of the gamma-ray and X-ray emission suggest a rather low value of the magnetic field strength downstream the shock which implies a rather weak radiation cooling which does not strongly modify the X-ray spectrum. In contrast the damping of the magnetic field can effectively soften the spectrum of the X-ray emission inside of the SNR.

Although magnetic field damping scenario can reproduce the trend of the spectral softening towards the interior of the remnant it fails to correctly reproduce the amplitude of the spectral variation. This discrepancy, however, might be simply a projection effect. In our simulations we use the assumption of a spherical symmetry, while in reality the Vela Jr. SNR is not spherically symmetric exhibiting a bright north western rim. If we assume that the emission from the remnat is dominated by a part of the shell limited by some solid angle $\Omega$ resulting radial profiles would not suffer from the projection of the emission from the interior of the remnant with a lower flux and softer spectrum. Therefore, resulting simulated profiles would much better resemble the observational data.

\section{References}

Aharonian, F., Akhperjanian, A. G., Bazer-Bachi, A. R., et al. 2007, ApJ, , 661, 236

Allen, G. E., Chow, K., DeLaney, T., et al. 2015, ApJ, , 798, 82

Aschenbach, B. 1998, Nature, , 396, 141

Aschenbach, B., Iyudin, A. F., \& Schönfelder, V. 1999, A\&A, , 350, 997 
Bamba, A., Yamazaki, R., \& Hiraga, J. S. 2005, ApJ, , 632, 294

Berezhko, E. G., Pühlhofer, G., \& Völk, H. J. 2009, A\&A, , 505, 641

Blasi, P., Gabici, S., \& Vannoni, G. 2005, MNRAS, , 361, 907

Duncan, A. R., \& Green, D. A. 2000, A\&A, , 364, 732

H. E. S. S. Collaboration, Abdalla, H., Abramowski, A., et al. 2016, ArXiv e-prints, arXiv:1611.01863

Iyudin, A. F., Pakhomov, Y. V., Chugai, N. N., et al. 2010, A\&A, , 519, A86

Katsuda, S., Tsunemi, H., \& Mori, K. 2008, ApJ, , 678, L35

Kishishita, T., Hiraga, J., \& Uchiyama, Y. 2013, A\&A, , 551, A132

Mignone, A., Zanni, C., Tzeferacos, P., et al. 2012, ApJS, , 198, 7

Pohl, M., Wilhelm, A., \& Telezhinsky, I. 2015, A\&A, , 574, A43

Pohl, M., Yan, H., \& Lazarian, A. 2005, ApJ, , 626, L101

Rettig, R., \& Pohl, M. 2012, A\&A, , 545, A47

Reynoso, E. M., Dubner, G., Giacani, E., Johnston, S., \& Green, A. J. 2006, A\&A, , 449, 243

Slane, P., Hughes, J. P., Edgar, R. J., et al. 2001, ApJ, , 548, 814

Tanaka, T., Allafort, A., Ballet, J., et al. 2011, ApJ, , 740, L51

Telezhinsky, I., Dwarkadas, V. V., \& Pohl, M. 2012, Astroparticle Physics, 35, 300

-. 2013, A\&A, , 552, A102 

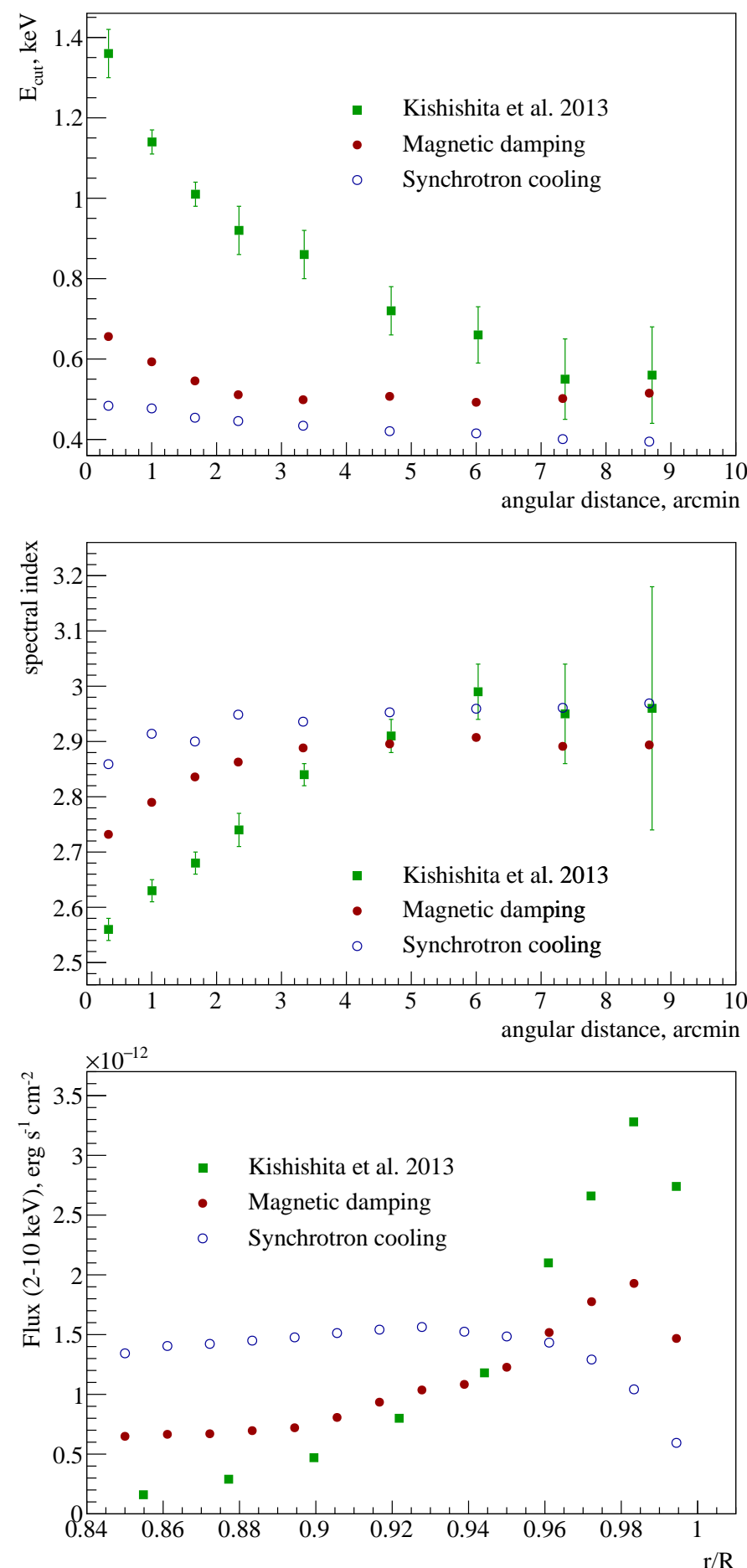

Figure 2: Simulated X-ray radial profiles for the magnetic field damping (filled red circles) and synchrotron cooling (empty blue circles) scenarios. Top and middle panels show the cut-off energy and the spectral index respectively as a function of the angular distance from the SNR shock. Bottom plot shows the $2-10 \mathrm{keV}$ flux as a function of the distance from the center of the shock in the units of the shock radius. In all plots green squares indicate the observational data (Kishishita et al., 2013). 